\title{
RESILIENCE OF AIR TRAFFIC CONTROLLERS IN CONTROL TOWER
}

\author{
Daisuke Karikawa ${ }^{1}$ \\ Hisae Aoyama ${ }^{2}$ \\ Tomoki Ohashi ${ }^{3}$ \\ Makoto Takahashi ${ }^{1}$ \\ Masaharu Kitamura ${ }^{4}$ \\ 1) Tohoku University, Japan \\ 2) National Institute of Maritime, Port and Aviation Technology, Japan \\ 3) Miyagi Gakuin Women's University, Japan \\ 4) Research Institute for Technology Management Strategy Co. Ltd., Japan
}

\begin{abstract}
The air traffic controllers in the control tower at an airport are responsible for the safe and efficient movements of aircraft on the taxiways and runways of the airport, and of aircraft in the airspace near the airport. In aerodrome Air Traffic Control (ATC), controllers always face inevitable disturbances, such as changing traffic situations, variability in the performance of pilots, variable wind conditions, and so on. However, controllers deal with these challenging situations to provide safe and efficient ATC services continuously. The aim of this study is to determine the resilience of controllers during their normal daily ATC operations. Multiple interviews with tower controllers and observations in the control tower at Chubu Centrair International Airport in Japan were conducted to investigate their working processes, including cognitive aspects. Their answers to the interview questions were analyzed from the perspective of Safety-II, that is, how they manage disturbances in their working environment to achieve successful ATC operations. The analysis results are described using the functional resonance analysis method (FRAM), modified for the description of three types of functions and situational factors: (1) basic functions for meeting the separation standards between aircraft, (2) threat factors, which could disturb the basic functions, and (3) response functions for managing possible negative effects caused by the threat factors. The FRAM model obtained visualizes the complex interactions among the functions and the situational factors in aerodrome ATC tasks, and it shows the essential role of performance adjustments of controllers for the proper functioning of ATC.
\end{abstract}

Keywords: Safety-II, FRAM, Air Traffic Control

\section{INTRODUCTION}

In contrast to the traditional safety perspective (Safety-I), which focuses only on things that can go wrong to eliminate the causes of failure, Safety-II also focuses on things that can go right to ensure and promote success. In other words, one of the essential elements of safety management

(C) 2019 Authors. This is an Open Access article distributed under the terms of the Creative Commons AttributionNonCommercial 4.0 International License (http://creativecommons.org/licenses/by-nc/4.0), permitting all non-commercial use, distribution, and reproduction in any medium, provided the original work is properly cited.

ISBN: 978-91-88898-41-8

DOI: https://doi.org/10.15626/rea8.16 
based on a Safety-II perspective is learning from acceptable outcomes and acceptable performance in normal operations.[1] However, there are three outstanding issues for safety management in the practical implementation of the lessons learned from success in everyday work. The first issue is that it is very difficult for highly skilled practitioners to explain how they achieve and maintain success (for example, how they identify potential disturbances and how they prepare and manage these in real work settings) in a way that safety managers and researchers, who may not have the experience of a skilled practitioner, can understand. This can lead to difficulties in extracting the factors contributing to success. The second issue is identifying appropriate visualization methods to describe the factors contributing to success so that the personnel responsible for safety management can share their understanding of the contributing factors and their effects. The third issue is developing concrete applications of the lessons learned through analyses of normal operations to promote success and enhance safety.

The authors have conducted field research of aerodrome Air Traffic Control (ATC) tasks to address the issues noted above with the cooperation of air traffic controllers at Chubu Centrair International Airport (Chubu Airport). ATC is defined as "a service provided for the purpose of preventing collisions between aircraft, and on the maneuvering area between aircraft and obstructions; and expediting and maintaining an orderly flow of air traffic."[2] It is studied in resilience engineering[3] and known as a typical example of a resilient system. In ATC tasks, controllers achieve safe and efficient air traffic flow whilst working in a changing environment, such as continuously changing traffic situations, weather conditions, and so on. In short, ATC tasks require resilient performance, not only in emergency responses but also in normal daily operations.

The aim of the present paper is to analyze and model controllers' resilience in achieving stable ATC services under varying conditions. Multiple interviews with tower controllers and observations in the control tower at Chubu Airport were conducted to investigate their working processes, including cognitive aspects. Their answers to the interview questions were analyzed from the perspective of Safety-II, that is, how they can manage variable conditions and disturbances in their working environments to achieve successful ATC operations. The analysis results were described using the Functional Resonance Analysis Method (FRAM) [4].

\section{AERODROME AIR TRAFFIC CONTROL}

An aerodrome ATC service is responsible for the safe and efficient movement of aircraft on the taxiways and runways at the airport, and for the safety of aircraft in the airspace near the airport. The aerodrome ATC service of Chubu Airport, the airport studied in this research, is provided by four controllers assigned to different functions: Clearance Delivery (CD), Flight Data (FD), Ground Controller (GC), and Local Controller (LC). The role of the CD is to deliver route clearances to departing aircraft on the apron in response to clearance requests from pilots. The FD coordinates with other ATC facilities or divisions such as area control centers, the radar room (which provides a terminal control service), and related organizations, like the airport operations center. The GC is responsible for aircraft movements on aprons and taxiways. The $\mathrm{LC}$ is responsible for safety on the runway and for aircraft in the air by ensuring the prescribed separation is maintained between aircraft. 
As an example, Figure 1 shows a typical ATC procedure for an aircraft departure. The pilot of an aircraft on the apron requests route clearance from the CD (Figure 1(a)). The CD delivers the route clearance to the pilot if appropriate (before that, the FD may request route clearance from the area control center, if necessary). On receiving the route clearance, the pilot requests a pushback clearance from the GC (Figure 1(b)). Once the clearance is given by the GC, the aircraft is pushed backwards away from the gate by a special vehicle called a pushback tractor. When the pushback has finished, the pilot requests taxi clearance from the GC (Figure 1(c)).

Once that is received, the aircraft taxis to the departure runway via the designated taxiways. Responsibility for the aircraft is handed from the GC to the LC near the runway. Finally, the aircraft takes off after receiving takeoff clearance from the LC (Figure 1(d)).

For an arrival, the aircraft lands after receiving landing clearance from the LC, followed by taxing from the runway to a designated gate according to the taxi clearance issued by the GC. As described above, the four controllers cooperate to provide ATC services.

\begin{tabular}{|c|c|}
\hline Pilot & Controller \\
\hline Requests route clearance (a) $>>>$ & $\begin{array}{l}\text { Clearance Delivery (CD) } \\
\text { Receives route clearance from the area control } \\
\text { center via the FD (if necessary) } \\
<<\text { Delivers the route clearance }\end{array}$ \\
\hline $\begin{array}{l}\text { Requests pushback clearance }(\mathrm{b})>>> \\
\text { Reads back the received clearance }>>> \\
\text { (starts pushback) }\end{array}$ & $\begin{array}{l}\text { Ground Controller (GC) } \\
<<<\text { Issues the pushback clearance } \\
\text { Checks whether the read-back is correct }\end{array}$ \\
\hline $\begin{array}{l}\text { Requests taxi clearance }(\mathrm{c})>>> \\
\text { Reads back the received clearance }>>> \\
\qquad \text { (starts taxiing) } \\
\text { Reads back the received instruction }>>>\end{array}$ & $\begin{array}{l}<<<\text { Issues taxi clearance } \\
\text { Checks whether the read-back is correct } \\
<<<\text { Issues instructions of radio contact to local } \\
\text { controller (LC) } \\
\text { Checks whether the read-back is correct }\end{array}$ \\
\hline $\begin{array}{l}\text { Radio contact to LC } \gg>> \\
\text { Reports ready to take off }>>> \\
\text { Reads back the received clearance }>>> \\
\text { (take offs) }\end{array}$ & $\begin{array}{l}\text { Local Controller (LC) } \\
<<<\text { Response } \\
<<<\text { Confirms the aircraft is ready to take off } \\
<<<\text { Issues takeoff clearance (d) } \\
\text { Checks whether the read-back is correct }\end{array}$ \\
\hline
\end{tabular}

Figure 1. Example of ATC process for an aircraft departure 


\section{METHOD}

The first step in starting a task analysis is to choose reasonable methods and tools for the data collection and analysis. For aerodrome ATC, it is difficult to gather and analyze all the objective data used by controllers in making decisions, partly because they use multiple sources of information, including visual observations from the control tower. A tower simulator, which can be used by researchers to reproduce the same situations and scenes for analyses, is a possible useful tool for mitigating this difficulty. However, even full-scale tower simulators have still a deficiency in reproducing all of the important information sources for controllers with high fidelity, such as the movement of various vehicles, ground staff, and passengers.

The present research, therefore, adopted interviews with the controllers as the primary method of data collection for the task analysis, supplemented by field observations, although this method obviously has limitations too. The number of participants and the length of the interviews varied, because they were conducted over five years. In general, there were three or four controllers in each interview, which lasted for an hour or two.

The questions in the first few times of the interviews focused on the basic procedures of four controller positions (CD, FD, GC and LC) for aircraft departure and arrival. The participant controllers were asked what information they needed to perform their tasks and the sources of this information. The answers gave the authors a fundamental understanding of aerodrome ATC tasks. Field observations at the control tower were conducted to deepen the authors' understanding of the procedures. Following these careful preliminary investigations, the interviews proceeded to the next stage, which addresses a key question of this research: how do the controllers manage disturbances in their working environment to achieve successful ATC operations? Although concrete interview methods for effectively extracting answers to the key question are still being explored, interviews for investigating the question probably need to include questions to identify:

1) Basic functions to achieve successful operations. These are functions such that, if they do work inadequately, successful operations are not achieved, regardless of the situation and conditions.

2) Threat or disturbance factors that can disturb the proper functioning of the basic functions by imposing potential negative effects on them, such as time limitations, resource constraints, and degradation of control.

3) Controllers' preparations and responses to prevent or mitigate the negative effects due to the disturbance factors.

\section{RESULTS}

The findings from the interviews were analyzed based on the basic principles of the FRAM. Figure 2 shows the FRAM model developed in the present study for functions relating to the primary task of the LC, that is, to ensure the prescribed separation between aircraft.

The green hexagons present the basic functions of the separation assurance task. Functions $<$ monitoring the traffic $>$, $<$ recognizing a target aircraft that requires intervention by a controller $>$, < planning and issuing instructions or clearances $>$, and $<$ recognizing the time window has opened for issuing instructions $>$ are classified as basic functions. Monitoring whether the change of state of an aircraft resulting from ATC instructions is consistent with the controller's intention $(<$ monitoring the change of aircraft state $>)$ is also classified as a basic function. Whilst 
these basic functions are a simplified form of the real ATC tasks, which are much more complex, they are suitable for considering the main research question.

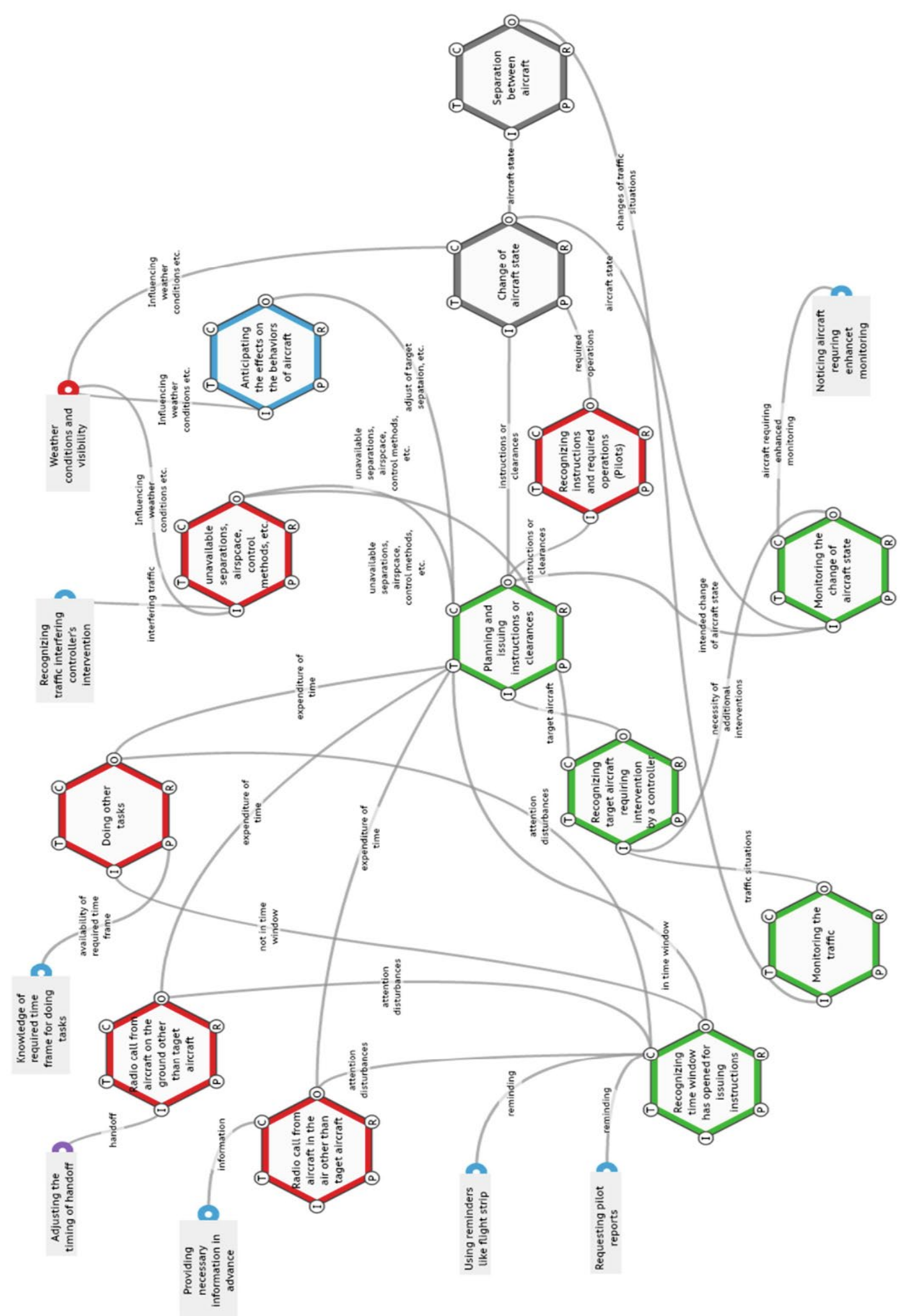

Figure 2. FRAM model for ensuring aircraft separation by the local controller 
The red hexagons are the threat nodes, which correspond to threat factors that could have negative effects on the basic functions. Although the threat factors are inevitable in an aerodrome ATC (such as <weather conditions and visibility $>$ ) or necessary activities (such as $<$ doing other tasks $>$ ), they can also work as potential causes of constraints or difficulty in performing the basic functions for the target aircraft. A typical example of a threat factor is a radio call from the pilot of an aircraft other than the target aircraft. Such calls are inevitable and often necessary from the perspective of the appropriate handling of the entire air traffic. However, if such a calls occurs when a controller is due to issue ATC instructions to the target aircraft, then performing the basic function <planning and issuing instructions or clearances> at the right time may be disturbed because the LC is obliged to make a response. It is a core skill for controllers to recognize or anticipate threat factors and their negative effects in providing safe, efficient, and stable ATC services. Therefore, the function <recognize or anticipate threat nodes $>$ is essential, although it is omitted in Fig. 2 to simplify the FRAM model.

The blue hexagons are the response function nodes, which represent a controller's specific activities to prevent or mitigate the negative effects of the threat nodes. The interviews revealed that controllers can recognize or anticipate potential disturbances, and adjust their performance such as planning, timing, and communications to deal with them. Threat nodes are categorized as uncontrollable threats and controllable threats. An example of the uncontrollable threats is $<$ weather and wind conditions $>$. Since it is obviously impossible to control the weather and wind conditions, the response functions for this threat node are characterized by mitigation measures. If controllers anticipate the effects of adverse weather and wind conditions on the behavior of aircraft (such as an increased frequency of go-arounds due to adverse wind conditions, extended runway occupation time due to snow, and so on), they can adopt control strategies with a larger safety margin. Uncontrollable threats are exemplified by a radio call from an aircraft other than the target aircraft. The number of such radio calls air can be, at least somewhat, reduced by providing necessary information, such as traffic information, to pilots in advance. In addition, to reduce the number of radio calls to the LC from other aircraft taxiing on the ground at times of high workloads, the GC can adjust the timing of the handoffs of aircraft to the LC (indicated by a violet hexagon). Thus, making adjustments to the tasks done by the controller team also plays an important role in maintaining successful ATC operations.

\section{DISCUSSION}

In the previous section, a modified descriptive method of a FRAM model with three types of nodes (basic function nodes, threat nodes, and response function nodes) was proposed. Using the descriptive method, a FRAM model that includes the functions and their interactions for accomplishing the separation assurance task of the LC was developed based on the findings from the interviews with tower controllers.

The FRAM model in Figure 2 strongly implies that noticing threat nodes and the preparations or responses of controllers against them are indispensable for accomplishing the separation assurance task. In addition, the FRAM model visualizes the complex interactions among the functions and relative situational factors, including time limitations, resource constraints, and degradation of control. That means that normal ATC operations cannot be achieved without fulfilling each of the functions shown in Figure 2 at an acceptable level. This indicates the importance of performance adjustments of controllers for the proper functioning of ATC.

Figure 2 also shows the features of the FRAM in analyzing and visualizing the complex interactions among the functions and situational factors. For the wider utilization of FRAM in safety management and other safety-related activities, it is necessary to demonstrate the 
practical benefits offered by the features of the FRAM. A potential benefit is that the FRAM model in Figure 2 allows explicit discussions about the effectiveness and reliability of response functions, which may lead to a better understanding of the resilience and brittleness of the system.

\section{SUMMARY}

The present research included multiple interviews with controllers. It has revealed the controllers' resilience in achieving stable ATC services even in variable conditions. To model the controllers' resilient performance, a modified descriptive method of a FRAM model with three types of nodes (basic function nodes, threat nodes, and response function nodes) was proposed. The developed FRAM model, which depicts a subset of the aerodrome ATC tasks, clearly shows the complex interactions among the functions and situational factors, like the impositions of time limitations, resource constraints, and degradation of control. The FRAM model also strongly implies that noticing threat nodes and the preparation or responses of controllers against them are indispensable for managing such complex interactions, including the negative effects and proper functioning of ATC operations.

Our future work includes developing interview methods to identify the factors contributing to success in normal operations, asking the controllers to evaluate the developed FRAM model, and exploring the practical application of FRAM in safety management and other safety- related activities.

\section{ACKNOWLEDGEMENTS}

The authors would like to thank the air traffic controllers of Chubu Centrair International Airport in Japan and West Japan Civil Aviation Bureau for understanding and cooperation in multiple interviews and field observations for five years. This work was supported by JSPS KAKENHI (grant JP17H02045).

\section{REFERENCES}

[1] Hollnagel,E.(2014).Safety-IandSafety-II,AshgatePublishing.

[2] International Civil Aviation Organization (ICAO). (2018). Annex 11 to the Convention on International Civil Aviation, Air traffic Services, $15^{\text {th }}$ Edition.

[3] Malakis,S.,andKontogiannis,T.(2010).CognitiveStrategiesinEmergencyand Abnormal Situations Training-Implications for Resilience in Air Traffic Control. In Hollnagel, E., Paries, J., Woods, DD., and Wreathall, J. (Eds.) Resilience Engineering in Practice-A Guidebook, Ashgate Publishing, pp. 101-117.

[4] Hollnagel,E.(2012).FRAM:TheFunctionalResonanceAnalysisMethod:Modelling Complex Socio-technical Systems, CRC Press. 